\title{
Identificación de perfiles ambientales en la Pyme a través de la auditoría ambiental
}

Fabiola Maribel

Denegri de Dios

Universidad Autónoma de

Baja California. fdenegri@

uabc.edu.mx

\section{César Ángel Peña}

Salmón

Universidad Autónoma de Baja California. cesarpena@ uabc.mx, cepesa@hotmail.com

\section{Resumen}

Las micro, pequeñas y medianas empresas (Pyme) han sido consideradas como un grupo objetivo secundario de las políticas de control de contaminación en México. Aunque su impacto ambiental individual puede ser pequeño, su impacto agregado puede causar serios problemas para el ambiente y la población. Recientemente, en México se han implementando programas de autorregulación ambiental dirigidos a Pyme utilizando como herramienta principal la auditoría ambiental. El propósito de este trabajo es identificar los perfiles de contaminación y cumplimiento ambiental de las Pyme que han participado en el Programa de Autorregulación Ambiental en Mexicali, Baja California. Los datos utilizados se obtuvieron de los informes de auditoría ambiental de aproximadamente sesenta Pyme que participaron en el programa en los años 2005 y 2006. Los resultados muestran que tres de las ramas participantes obtuvieron los perfiles de contaminación más altos con perfiles de cumplimiento ambiental casi nulos.

Palabras clave: autorregulación ambiental, cumplimiento ambiental, auditoría ambiental, Pyme, México. 


\title{
Identification of SMEs' environmental profiles through environmental audi- ting
}

\begin{abstract}
Small and medium enterprises (SMEs) have been considered as a secondary target group of pollution control policies in Mexico. Although their individual environmental impact may be small, their aggregate impact can cause serious problems for people and the environment. Recently, environmental self-regulation programs for SMEs have been implemented in Mexico, with the use of environmental auditing as a main tool. This work aims to identify pollution profiles and environmental compliance profiles of SMEs that participated in the Environmental Self-Regulation Program, in Mexicali, B.C., Mexico. Data were obtained of sixty environmental audit reports of SMEs participants in the program during 2005 and 2006. The results show that three of the industrial branches that participated in the program reached the highest pollution profiles with an almost null environmental compliance profile.
\end{abstract}

Keywords: environmental self-regulation, environmental compliance, environmental auditing, SMEs, Mexico.

\section{Introducción}

Desde el punto de vista económico, las Pyme son un segmento fundamental en la economía de diversos países; la OCDE afirma que en sus países miembros las Pyme representan más del $95 \%$ de las empresas, generan dos terceras partes de los empleos y son la fuente principal de nuevos empleos (OCDE, 2005). En México, en el año 2003, las Pyme contribuyeron con el $99.8 \%$ de las empresas y el $73 \%$ de los empleos en el país (INEGI, 2004). Pero ¿qué significan estos datos en términos ambientales?

Desde el punto de vista ambiental, actualmente existe una escasa disponibilidad de información objetiva que dé cuenta de forma precisa sobre la contaminación total ${ }^{1}$

\footnotetext{
${ }^{1}$ En países como el Reino Unido se ha estimado que las Pyme pueden generar el 70\% del total de la contaminación industrial (Gunningham, 2002; Hillary, 1995); en la India se estima que generan el 65\% de los residuos industriales (UNEP, 2003); en un estudio en países asiáticos se llegó a la conclusión de que si bien las Pyme pueden no ser responsables de la mayor parte del deterioro ambiental, probablemente son contaminadoras más graves por unidad de producción que las grandes empresas (Kent, 1991)
} 
que generan las Pyme en la mayoría de los países desarrollados o en desarrollo (Brío y Junquera, 2002; Domínguez, 2003; Tilley, 1999), por lo que un paso fundamental para el establecimiento de estrategias ambientales para estas empresas es identificar los niveles de contaminación y el cumplimiento ambiental que presentan en un mercado local específico.

En los últimos años, la importancia del control de la contaminación y del cumplimiento ambiental de las Pyme se ha reflejado en la agenda de diferentes organismos y países; por ejemplo, la Agenda 21 para el desarrollo sustentable señala la necesidad de fomentar la utilización de sistemas limpios de producción con especial énfasis en las Pyme (ONU, 1992); también se ha manifestado en un aumento de iniciativas como el programa de la Comisión Europea Small, Clean and Competitive y el proyecto Competitividad y Medio Ambiente (CYMA) del Mercosur.

Existen diversos instrumentos en materia ambiental que pueden ser adaptados y aplicados para lograr mejoras en la evaluación de la contaminación y el cumplimiento ambiental de las Pyme (Parker y cols., 2009; Romo, 2005; Starkey, 1998), entre ellos, la auditoría ambiental, que permite examinar — entre otros aspectosel tipo de emisiones contaminantes que generan, el grado de cumplimiento ambiental que presentan y las acciones o planes correctivos necesarios para mejorar el cumplimiento o desempeño ambiental (Bojórquez-Tapia, 1995; Carmona, 1995; Hillary, 2000; Starkey, 1998); sin embargo, en México, este instrumento había sido dirigido principalmente a las grandes industrias. Recientemente, esto cambió con el impulso a los programas de autorregulación ambiental locales dirigidos a las Pyme, como es el caso del Programa de Autorregulación Ambiental (PAA) aplicado en la ciudad Mexicali, Baja California.

El propósito de este trabajo es identificar los perfiles de contaminación y cumplimiento ambiental de las Pyme participantes en el PAA en Mexicali, B. C. durante los años 2005 y 2006; para ello, se utilizaron los informes de auditoría ambiental (AA) de aproximadamente 60 empresas. Los resultados muestran, por un lado, que la mayoría de las Pyme presentan un perfil de cumplimiento ambiental nulo; por otro, que existen distintos perfiles de contaminación entre las ramas participantes destacando tres ramas con un perfil de contaminación muy alto.

El documento está estructurado en seis partes: 1) factores que condicionan el cumplimiento ambiental de las Pyme, 2) los instrumentos e iniciativas para mejorar el cumplimiento ambiental de las Pyme, 3) la auditoría ambiental y el Programa de 
Autorregulación Ambiental en Mexicali, B. C., 4) la descripción de la metodología utilizada, 5) la presentación de los resultados obtenidos y 6) se presentan las principales conclusiones.

\section{Factores que condicionan el cumplimiento ambiental de las Pyme}

En la literatura revisada se encontraron diversos tipos de respuestas al cumplimiento ambiental por parte de las Pyme: desde las respuestas más reactivas que tienen como meta exclusiva cumplir con la legislación ambiental hasta las respuestas más proactivas que perciben el cuidado del ambiente más como una fuente de oportunidades que como un problema a afrontar al mínimo costo (Brío y Junquera, 2002; Tilley, 1999). Sin embargo, en diversos estudios empíricos se constata que la mayoría de las Pyme, aun cuando las presiones externas se endurecen progresivamente, siguen presentando una respuesta reactiva de mero cumplimiento o en su caso una estrategia de resistencia o de no cumplir ambientalmente (Brío y Junquera, 2002; Gunningham, 2002; Parker y cols., 2009; Tilley, 1999).

Lo anterior sitúa al mayor número de Pyme, tanto de los países desarrollados como en desarrollo, en un nivel bajo de desempeño y/o cumplimiento ambiental con respecto a las grandes empresas (Hillary, 2000; Revell, 2002). Esto ha generado un aumento en la literatura que identifican los factores que limitan el cumplimiento ambiental de este tipo de empresas, entre los cuales podemos enlistar los siguientes:

a) Procesos menos eficientes ambientalmente, pues utilizan técnicas de producción que requieren de un mayor uso de recursos y generan mayor contaminación por unidad de producto (Cepis, EPA y BID, 2000).

b) Un estilo directivo con escasa formación ambiental (conocimiento técnico y jurídico), carencia de conciencia ambiental del impacto ambiental de su empresa, así como una orientación a corto plazo de los dueños y/o gerentes (Brío y Junquera, 2002; Domínguez, 2003; Gunningham, 2002; Meritt, 1998; Revell, 2002, Parker y cols., 2009).

c) Una débil estructura organizativa (organización mal estructurada y actividades no estandarizadas) que dificulta la implementación de mejoras ambientales (Brío y Junquera, 2002).

d) Carecen de capacidad y medios financieros para absorber los costos de controlar o reducir las emisiones que generan (Brío y Junquera, 2002; Cepis, 
EPA y BID, 2000; Domínguez, 2003; Hillary, 2000); además, perciben el cumplimiento ambiental como un costo que disminuye sus ganancias (Gunninham, 2002; Revell, 2002).

e) Poseen menor acceso a tecnología, diseño y control de procesos que las grandes empresas (Brío y Junquera, 2002; Cepis, EPA y BID, 2000).

f) Presentan una menor capacidad para realizar innovaciones que las grandes empresas (Brío y Junquera, 2002).

g) Las regulaciones ambientales benefician más a las grandes empresas que a las pequeñas (Brío y Junquera, 2002).

h) El control y vigilancia del cumplimiento ambiental de las Pyme resulta difícil para las autoridades correspondientes debido a que son numerosas, abarcan diversidad de productos y procesos, y se distribuyen en forma dispersa en las localidades (Cepis, EPA y BID, 2000; Gunningham, 2002).

i) La falta de presiones o demandas externas de cumplimiento ambiental, por ejemplo, por parte de sus clientes en cadenas productivas o por parte de las autoridades competentes (Gunningham, 2002; Parker y cols., 2009).

La combinación de estos factores le da a cada Pyme un nivel de respuesta de cumplimiento ambiental y la suma o conjunto de niveles de cumplimiento ambiental del total de Pyme localizadas en una ciudad o lugar determinado pueden - de acuerdo con el tipo y nivel de contaminación - traducirse en afectaciones graves para el ambiente y la población. Lo anterior ha fomentado, en los últimos años, un creciente interés por el análisis de los instrumentos e iniciativas disponibles para mejorar el cumplimiento y desempeño ambiental de las Pyme. En el próximo apartado se revisan diversos instrumentos e iniciativas para mejorar el cumplimiento ambiental de estas empresas.

\section{Instrumentos e iniciativas para mejorar el cumplimiento ambiental de las Pyme}

Romo (2005) señala que las autoridades ambientales carecen de la capacidad para el monitoreo del cumplimiento ambiental del total de las Pyme, por lo que ciertos instrumentos no son aplicables a este sector (p. e. estándares de emisiones); por 
tanto, sugiere los siguientes instrumentos para el caso de dichas empresas: a) de comando y control, los estándares de tecnología, la relocalización de plantas; b) incentivos económicos, los impuestos a insumos o productos contaminantes, subsidios a insumos o productos limpios, precios y tarifas de bienes y servicios del sector público, incentivos fiscales, esquemas de depósito-reembolso; c) inversión del gobierno, la construcción de infraestructura ambiental para el tratamiento de desperdicios; d) basados en información y esquemas voluntarios, desarrollo de tecnologías, programas para dar a conocer públicamente el desempeño ambiental de las empresas, programas educativos y acuerdos voluntarios.

Starkey (1998) reconoce como herramientas de gestión ambiental para las Pyme los sistemas de gestión ambiental (SGA), la auditoría ambiental, los indicadores ambientales, el análisis de ciclo de vida, el ecoetiquetado ambiental, el establecimiento de una política ambiental de la empresa, el ecobalance y el reporte ambiental.

Gunningham (2002) identifica como instrumentos capaces de influir en la actitud y comportamiento ambiental de las Pyme la educación y capacitación ${ }^{2}$; la autoinspección y autoauditoría; la implementación de Sistemas de Gestión Ambiental (ISO-14000, EMAS); considerar las relaciones entre clientes y proveedores; la corregulación de la industria ${ }^{3}$, la aplicación de incentivos económicos. El autor precisa la necesidad de considerar las características específicas de los diferentes grupos de Pyme; también advierte que las regulaciones ambientales son el factor más influyente para que dichas empresas determinen una estrategia de gestión ambiental, pues en ausencia de regulaciones, la experiencia sugiere que la aplicación de otros instrumentos falla, aun cuando sea racional su aplicación.

Una de las iniciativas gubernamentales más utilizada es la disposición de información, asesoría y líneas de ayuda directa o indirecta, lo cual incluye contar con una página en Internet o una línea telefónica que provee información sobre la legislación ambiental y la gestión ambiental, sobre opciones de financiamiento, folletos que informan sobre las modificaciones y actualizaciones de las regulaciones ambientales, también incluye la disponibilidad de asesores ambientales directos, etc. (Monkhouse y cols., 2006; Parker y cols., 2009).

\footnotetext{
${ }^{2}$ En algunos países la capacitación incluye a los empresarios, empleados del sector público y estudiantes que trabajan en conjunto para conseguir ciertas metas, por ejemplo, la certificación ambiental de una Pyme (Monkhouse y cols., 2006).

${ }^{3}$ La corregulación de la industria se da a través de la transferencia de la responsabilidad de administrar la legislación y las regulaciones de los gobiernos a las industrias (Gunningham, 2002).
} 
El soporte financiero o financiamiento es otro tipo de iniciativa que puede establecerse en forma de subvenciones, préstamos, privilegios tributarios, créditos con interés preferente, etc. Un estudio de Pimenova y van der Vorst (2004) muestra el soporte financiero como el segundo elemento que favorece la mejoras ambientales en las Pyme, después de la información y asesoría.

Las iniciativas con un enfoque de redes consideran que las Pyme son particularmente dependientes de las redes de las que forman parte; por lo tanto, pueden ser usadas para transmitir conocimiento e información sobre mejores prácticas, innovaciones, etc. Algunos ejemplos de estas iniciativas son la International Network for SMEs en Italia, Green Network en Dinamarca y la Finnish-Estonian Environmental Networking en Finlandia (Monkhouse y cols., 2006; UNEP, 2003). Aquí, pueden incluirse las iniciativas con sistemas de soporte de mentores donde empresas grandes apoyan en la gestión ambiental de las Pyme que forman parte de su cadena de proveedores, un ejemplo de este tipo de iniciativas fue el Guadalajara Environmental Management Pilot implementado en México en 1996 (Banco Mundial, 1998).

Los acuerdos voluntarios o negociados entre asociaciones de empresas y el gobierno permiten trabajar hacia objetivos ambientales específicos y evitar la amenaza inmediata de regulaciones ambientales directas. Algunos ejemplos internacionales son los Acuerdos Holandeses, el Acuerdo Ambiental Bávaro en Alemania, el Plan de Acción Voluntaria Keidanren en Japón (Monkhouse y cols., 2006).

Otras iniciativas dirigidas a las Pyme son la ecoeficiencia, la producción más limpia y la prevención de la contaminación, las cuales se caracterizan por un enfoque de estrategia ambiental preventiva a diferencia de estrategias de final del tubo; esto implica cambiar hacia un enfoque de atacar las causas de la contaminación, lo que incluye rediseño de productos, cambio de insumos, cambio y/o modificaciones de procesos, etc. (INE y cols., 2000; Hobbs, 2000).

En el caso de México, organismos internacionales como GEMI (Global Environmental Management Initiative) y GTZ (Agencia de Cooperación Técnica Alemana) y nacionales como los CMP+L (Centros de Producción más Limpia) ofrecen listados de autoverificación o autodiagnóstico ambiental, guías de autoevaluación ambiental, que pueden ser utilizados por las Pyme. Una de las limitaciones de las iniciativas mexicanas es su cobertura geográfica, como es el caso del CMP+L, 
pues está localizado en el centro del país, lo cual provocó que recientemente se instalaran dos centros regionales en los estados de Tabasco y Chihuahua.

La Fundación Mexicana para la Innovación y Transferencia de Tecnología (Funtec) y la Comisión para la Cooperación Ambiental de América del Norte (CCA) crearon el Fondo para Proyectos de Prevención de la Contaminación (Fiprev) para apoyar a las Pyme mexicanas en la realización de inversiones y transferencia de tecnología a través de financiamiento y asesoría (Domínguez, 2003).

Como se puede observar, hay diferentes tipos de instrumentos para mejorar el cumplimiento ambiental de las Pyme; sin embargo, como señalan algunos autores, encontrar la mejor combinación para obtener una estrategia efectiva, con un enfoque win-win, no es una tarea fácil, pues depende de la capacidad para integrar los diversos factores que influyen en el comportamiento ambiental de las distintas Pyme que integran un mercado local y las capacidades de las autoridades ambientales para llevarla a cabo (Gunningham, 2002; Monkhouse y cols., 2006; Romo, 2005).

Un paso inicial en el diseño de estrategias e iniciativas ambientales efectivas consiste en tener un diagnóstico del nivel de contaminación y cumplimiento ambiental de las Pyme en cuestión; en este sentido, como veremos en el próximo apartado, la auditoría ambiental posee características que la hacen un instrumento de factible aplicación para tal propósito.

\section{La auditoría ambiental y el programa de autorregulación ambiental en Mexicali, B. C.}

Aunque el término auditoría no es nuevo, pues es ampliamente conocido por ser un instrumento de control interno o externo de la contabilidad empresarial, la utilización del término "auditoría ambiental" sí es relativamente reciente y comparte con el anterior los principios de control y verificación del cumplimiento de reglas o normas, pero aplicables al tema ambiental. La auditoría ambiental (AA) fue aplicada inicialmente en los Estados Unidos a finales de los años setenta entre los sectores ambientales que, como la industria química, estaban más involucrados en la afectación del ambiente y como respuesta al aumento de las leyes y regulaciones ambientales (Sánchez, 2002).

Para Gunningham (2002), dado los limitados recursos de las autoridades ambientales y el limitado alcance de muchas estrategias regulatorias convencionales, hay 
una necesidad de cambiar de la regulación directa hacia una variedad de estrategias alternativas, incluyendo el cumplimiento voluntario, la autoevaluación y el uso de terceros agentes como reguladores subrogados. En este contexto, instrumentos voluntarios como la autoauditoría ambiental son más apropiados para mejorar el desempeño ambiental de las Pyme.

El Art. 38 bis de la Ley General de Equilibrio Ecológico y Protección Ambiental (LGEEPA) define la auditoría ambiental de la siguiente manera:

Los responsables del funcionamiento de una empresa podrán en forma voluntaria, a través de la auditoría ambiental, realizar el examen metodológico de sus operaciones, respecto de la contaminación y el riesgo que generan, así como el grado de cumplimiento de la normatividad ambiental y de los parámetros internacionales y de buenas prácticas de operación e ingeniería aplicables, con el objeto de definir las medidas preventivas y correctivas necesarias para proteger el medio ambiente.

De acuerdo con Carmona (1995), la importancia de la AA radica en su gran utilidad como instrumento para identificar las necesidades y deficiencias de las industrias en materia ambiental como paso previo o comprobación posterior del establecimiento de un plan de gestión ambiental.

En México, la autorregulación y la AA fueron incorporadas en 1996 en la LGEEPA como instrumentos de política ambiental. Sin embargo, la AA fue inicialmente aplicada en grandes industrias altamente contaminantes o de alto riesgo ambiental que corresponden a la jurisdicción federal a través del denominado Programa Nacional de Auditoría Ambiental (Bojórquez-Tapia y García, 1995; INEGI y cols., 2000). No obstante, en los últimos años, diversos gobiernos estatales han puesto en marcha programas de autorregulación como es el caso de Guanajuato (Programa de Auditoría Ambiental), Michoacán (Programa de Auditoría Ambiental Estatal) y Baja California (Programa de Autorregulación Ambiental).

En Mexicali, fue en el año 2005 cuando se firmó un acuerdo entre la Dirección General de Ecología de Baja California ${ }^{4}$ y la Cámara Nacional de la Industria de la Transformación (Canacintra) con el propósito de fomentar y promover el cumplimiento ambiental de las Pyme a través del denominado Programa de Autorregulación Ambiental (PAA).

${ }^{4}$ Actualmente la Secretaría de Protección al Ambiente o SPA 
La auditoría ambiental (AA) aplicada en el PAA incluye la verificación del cumplimiento de la Ley de Protección al Ambiente para el Estado de Baja California ${ }^{5}$, del Reglamento de la Ley del Equilibrio Ecológico y Protección al Ambiente del Estado de Baja California en materia de Prevención y Control de la Contaminación del Agua, Suelo y la Atmósfera vigente desde 1993, del Reglamento de la Ley del Equilibrio Ecológico y Protección al Ambiente del Estado de Baja California en materia de Impacto Ambiental vigente desde 1992, de las normas oficiales mexicanas (NOM-085-SEMARNAT-1994, NOM-043-SEMARNAT-081-1994, NOM002-SEMARNAT-1996). En el caso de las NOM muchas Pyme no alcanzan los límites permisibles; sin embargo, se requieren estudios que así lo comprueben.

Parte de las actividades que desarrollan las Pyme fueron subsidiadas inicialmente con recursos del Programa de Apoyo a las Pyme de la Secretaría de Economía de Baja California y el Fideicomiso Empresarial de Mexicali (Fidem) a través de Canacintra (Firman plan, 2005). Las actividades subsidiadas incluyen el pago del auditor o asesor ambiental y los estudios ambientales de laboratorio (80\% Canacintra y 20\% empresario); el resto de los costos y gastos (compra de equipo, modificaciones de procesos e instalaciones) fueron absorbidos por cada Pyme. Otro incentivo para participar en el PAA fue la exención del 100\% del pago de derechos por trámites ante la Secretaría de Protección al Ambiente (Secretaría de Protección al Ambiente, 2006).

Durante el periodo 2005-2006, 16 empresas obtuvieron el reconocimiento de cumplimiento ambiental, cifra que aumentó a 39 empresas durante 2006-2007 (Secretaría de Protección al Ambiente, 2007). Pese al aumento en la participación para el siguiente periodo, se suspendió el PAA por cuestiones presupuestales (Secretaría de Planeación y Finanzas, 2008).

La AA permite examinar la situación ambiental de las Pyme de manera voluntaria; asimismo, ofrece un diagnóstico de la contaminación y el cumplimiento ambiental de dichas empresas. Estos datos no sólo son útiles para la gestión ambiental interna de las empresas, sino también para las autoridades ambientales al obtener los perfiles ambientales de las Pyme auditadas. Como señala Gunningham (2002), el uso de la AA requiere ser acompañada, por una parte, de incentivos económicos

\footnotetext{
${ }^{5}$ Antes de la Ley de Protección al Ambiente para el Estado de Baja California, vigente desde el año 2001, existía la Ley de Equilibrio Ecológico y Protección al Ambiente del Estado de Baja California.
} 
(carrots) y, por otra, de mayores presiones a través de mayor inspección o sanciones (sticks) para aquellas Pyme que no participan en este tipo de programas o no cumplen ambientalmente. En el caso del PAA, la AA ha sido acompañada de incentivos económicos (subsidios) que explican, en parte, la participación de las Pyme. En el siguiente apartado se describe la metodología utilizada para obtener los perfiles ambientales de las empresas participantes en el PAA.

\section{Metodología}

En este trabajo la definición de perfiles de contaminación (PP) y perfiles de cumplimiento ambiental (PC) de las Pyme está basada en el análisis de los informes de AA de aproximadamente sesenta empresas participantes en el PAA en Mexicali durante el periodo 2005-2006. La AA ofrece tres tipos de datos: 1) una lista de verificación del tipo de emisiones contaminantes generadas por la empresa a los distintos medios, 2) una lista de verificación del cumplimiento ambiental según la normatividad vigente y 3 ) un listado de las acciones o actividades necesarias para cumplir con las regulaciones y abatir o controlar las emisiones contaminantes.

Los tipos de datos uno y dos fueron los insumos para obtener los PP y PC. En primer lugar, a partir de la lista de verificación del tipo de emisiones contaminantes, se identificaron los PP por empresa. Los tipos de emisiones incluyen: en aire, óxidos de azufre $\left(\mathrm{SO}_{\mathrm{x}}\right)$, dióxido de azufre $\left(\mathrm{SO}_{2}\right)$, monóxido de carbono $(\mathrm{CO})$, partículas suspendidas totales (PST), óxidos de nitrógeno $\left(\mathrm{NO}_{\mathrm{x}}\right.$ ) y compuestos orgánicos volátiles (COV); en agua, incluye aguas residuales de proceso, metales pesados, carga orgánica y partículas sólidas suspendidas (PSS); otros rubros son el ruido (interno y externo) y los residuos peligrosos, no peligrosos y residuos sólidos municipales, aunque la normatividad estatal abarca sólo los no peligrosos.

En segundo lugar, a partir de la lista de verificación de los puntos cumplidos, se identificaron los perfiles de PC por empresa. Los rubros de cumplimiento ambiental incluyen documentos como el informe preventivo, autorización o manifiesto de impacto ambiental, estudio de riesgo, el registro de emisiones al aire y el registro de descarga de aguas residuales. El resto de los rubros de cumplimiento ambiental contenidos en el informe de la AA están clasificados en impacto ambiental, riesgo, emisiones al aire, COV, gases de combustión, PST, ruido, aguas residuales y residuos no peligrosos. 
Posteriormente, se establecieron cinco rangos para el PP y cinco rangos para el PC. Estos perfiles se definieron a partir de los criterios mostrados en el cuadro 1. Finalmente, se calculó el valor promedio del perfil de contaminación y del perfil de cumplimiento para cada rama.

\section{Cuadro 1}

Criterios para la definición de los perfiles contaminación y cumplimiento ambiental

\begin{tabular}{c|c|c|cc}
\hline $\begin{array}{c}\text { Tipo emisiones/ } \\
\text { empresa } \\
\%\end{array}$ & $\begin{array}{c}\text { Perfil } \\
\text { contaminación }\end{array}$ & $\begin{array}{c}\text { Puntos } \\
\text { cumplidos/ } \\
\text { empresa } \\
\%\end{array}$ & $\begin{array}{c}\text { Perfil } \\
\text { cumplimiento }\end{array}$ \\
\hline $0 \%$ & Pulo & $(0)$ & $0 \%$ & Pulo $(0)$ \\
\hline $1 \%-25 \%$ & Bajo $(1)$ & $1 \%-25 \%$ & Bajo $(1)$ \\
\hline $25 \%-50 \%$ & Medio (2) & $25 \%-50 \%$ & Medio (2) \\
\hline $50 \%-75 \%$ & Alto $(3)$ & $50 \%-75 \%$ & Alto $(3)$ \\
\hline $75 \%-100 \%$ & Muy Alto (4) & $75 \%-100$ & Muy alto (4) \\
\hline
\end{tabular}

\section{Resultados}

Las Pyme participantes en el PAA presentaron en promedio 23 empleados y poco más de la mitad (55\%) inició sus operaciones después del año 2000. Las ramas a las que pertenecen las Pyme participantes se agruparon en alimentos, bebidas, remanufactura o reconstructoras de piezas automotrices, productos metálicos, imprenta, papel, química, productos de cemento, madera, suministro de gas, recicladoras de residuos industriales, recicladoras de metal y servicio automotriz. Las cuatro últimas ramas pertenecen al sector servicios (16\%), el resto de las ramas pertenecen al sector industrial (84\%). En síntesis, las Pyme participantes en el PAA son, en su mayoría, micro y pequeñas industrias de creación relativamente reciente.

Los resultados muestran que tres ramas obtuvieron un PP muy alto las cuales fueron alimentos, remanufactura automotriz y bebidas. Las ramas de productos metálicos y suministro de gas obtuvieron un PP alto. En conjunto, estas cinco ramas representaron el 66\% del total de empresas participantes en el PAA. La rama con mayor participación en el PAA fue la de productos metálicos (ver cuadro 2). 


\section{Cuadro 2}

Perfil de contaminación y cumplimiento ambiental de ramas participantes en el PAA en Mexicali, B. C. 2005-2006

\begin{tabular}{|c|c|c|c|}
\hline Rama & $\begin{array}{c}\text { Perfil } \\
\text { Contaminación } \\
\text { (PP) }\end{array}$ & $\begin{array}{c}\text { Perfil de } \\
\text { Cumplimiento } \\
\text { (PC) }\end{array}$ & $\begin{array}{c}\text { Empresas } \\
\text { participantes } \\
(\%)\end{array}$ \\
\hline Alimentos & MUY ALTO & BAJO & 11 \\
\hline $\begin{array}{l}\text { Remanufactura } \\
\text { automotriz }\end{array}$ & MUY ALTO & NULO & 11 \\
\hline Bebidas & MUY ALTO & NULO & 2 \\
\hline $\begin{array}{l}\text { Productos } \\
\text { metálicos }\end{array}$ & ALTO & NULO & 40 \\
\hline Suministro de gas & ALTO & NULO & 2 \\
\hline $\begin{array}{l}\text { Productos de } \\
\text { cemento }\end{array}$ & MEDIO & NULO & 4 \\
\hline Imprenta & MEDIO & NULO & 5 \\
\hline Química & MEDIO & NULO & 2 \\
\hline Papel & MEDIO & NULO & 2 \\
\hline $\begin{array}{l}\text { Servicio } \\
\text { automotriz }\end{array}$ & MEDIO & NULO & 7 \\
\hline $\begin{array}{l}\text { Recicladora } \\
\text { residuos } \\
\text { industriales }\end{array}$ & MEDIO & NULO & 5 \\
\hline Madera & BAJO & BAJO & 7 \\
\hline Recicladoras metal & BAJO & NULO & 2 \\
\hline
\end{tabular}

Fuente: Elaboración propia

Como se puede observar en el cuadro 2, el PC predominante fue el nulo; es decir, la mayoría de las Pyme no cumplían con las regulaciones ambientales. Sin embargo, en los PP si hay diferencias importantes entre las ramas. La combinación de PP y PC por rama permitió identificar a las ramas de alimentos, remanufactura automotriz y productos metálicos del resto de las ramas al presentar un PP alto en combinación con un PC de bajo a nulo; estas ramas concentraron el mayor porcentaje de participación en el programa. De acuerdo con el Censo Económico (INEGI, 2004), la rama de alimentos y productos metálicos concentraban el mayor número de las Pyme en Mexicali, lo que las convierte en posibles focos de atención en el establecimiento de iniciativas ambientales futuras. A continuación se describen los perfiles de contaminación y de cumplimiento ambiental de las ramas con mayor participación en el PAA pertenecientes al sector industrial. 


\section{Productos metálicos}

En esta rama, las Pyme participantes en el PAA se dedicaban a la fabricación de todo tipo de piezas de metal. Sin embargo, se identificaron tres subgrupos de acuerdo con los procesos que realizan: 1) aquellas dedicadas a la galvanoplastia 2) aquellas que sólo transforman hierro y acero en piezas de metal sin acabado de pintura, proceso conocido como maquinado de piezas metálicas, y 3) aquellas que fabrican piezas de metal con acabado de pintura.

El perfil de contaminación que presentaron las empresas de galvanoplastia es muy alto e incluyeron emisiones al aire ( $\mathrm{NO}_{2}, \mathrm{CO}, \mathrm{PST}, \mathrm{COV}$, vapores, olores); aguas residuales potencialmente contaminantes; y generación de residuos peligrosos y no peligrosos. El cumplimiento ambiental de estas empresas, tanto en autorizaciones como en el resto de los rubros aplicables (emisiones a la atmósfera, aguas residuales y residuos peligrosos y no peligrosos), fue nulo.

Las empresas que fabricaban piezas de metal con acabado de pintura representaron el mayor porcentaje en la rama de productos metálicos (70\%). El PP de estas empresas fue alto e incluye la generación de emisiones al aire $\left(\mathrm{NO}_{2}, \mathrm{CO}, \mathrm{NO}_{x}, \mathrm{PST}\right.$, COV y humos de soldadura) y la generación de residuos no peligrosos y peligrosos. El cumplimiento ambiental que prevaleció en estas ramas fue nulo tanto en las autorizaciones como en el resto de los rubros ambientales aplicables (impacto ambiental, emisiones a la atmósfera y residuos no peligrosos).

Las empresas dedicadas al maquinado de piezas obtuvieron un perfil de contaminación bajo, caracterizado por emisiones a la atmósfera originadas de los humos de soldadura (PST) y residuos no peligrosos (rebaba o viruta metálica) derivados del proceso de corte y soldadura de piezas. El cumplimiento ambiental en este subgrupo fue nulo tanto en autorizaciones como en el resto de los rubros aplicables (impacto ambiental, emisiones a la atmósfera y residuos no peligrosos). En general, la fabricación de productos metálicos presentó un PP alto con un perfil de PC nulo.

\section{Alimentos}

En esta rama, las Pyme participantes en el PAA se dedicaban principalmente al procesamiento de hortalizas, frutas y frituras de maíz. Las empresas presentaron emisiones contaminantes en diversas etapas del proceso de producción e incluyeron emisiones al aire $\left(\mathrm{NO}_{2}, \mathrm{NO}_{\mathrm{x}}, \mathrm{CO}, \mathrm{So}_{\mathrm{x}}\right.$, gases de combustión, y vapores); aguas 
residuales con carga orgánica y partículas sólidas suspendidas; residuos no peligrosos como aceites residuales; y ruido. Además, en algunos casos, estas empresas fueron clasificadas como industrias riesgosas por manejar ciertas cantidades de sustancias peligrosas como el amoniaco.

En relación con el cumplimiento ambiental en materia de autorizaciones y registros, las empresas de esta rama presentaron el cumplimiento de alguna de las autorizaciones o registros. En el resto de los rubros aplicables esta rama mostró un bajo cumplimiento ambiental (impacto ambiental, riesgo, emisiones a la atmósfera, ruido, residuos no peligrosos y aguas residuales potencialmente contaminantes que superaron los límites permisibles según la NOM-001-ECOL-1996). Esta rama presentó un PP muy alto y un PC bajo.

\section{Remanufactura o reconstrucción de piezas para automóvil}

Esta rama incluye las Pyme dedicadas a la reconstrucción de piezas de automóviles como motores, transformadores, carburadores, alternadores, arranques. El proceso de producción de esta rama incluye el lavado de piezas con agua o solventes, desoldar la pieza, proceso de sand blast, ${ }^{6}$ pulido, barnizado, reconstrucción de la pieza, pintura, lijado, entre otros. Las Pyme de esta rama generan emisiones al aire $\left(\mathrm{NO}_{2}, \mathrm{So}_{\mathrm{x}}, \mathrm{COV}, \mathrm{PST}\right.$ y humos de soldadura); aguas residuales con carga orgánica y partículas sólidas suspendidas descargadas a la red de alcantarillado sin previo tratamiento; y residuos peligrosos. El cumplimiento ambiental que presentaron en materia de autorizaciones es nulo (autorización de impacto ambiental, registro de emisiones al aire, registro de aguas residuales), así como en el resto de los rubros aplicables (impacto ambiental, riesgo, emisiones a la atmósfera, ruido, residuos peligrosos y no peligrosos, y aguas residuales potencialmente contaminantes). En síntesis, esta rama presentó un PP muy alto y un PC nulo.

\section{Madera}

En esta rama, la mayoría de las Pyme participantes se dedicaban a la fabricación de tarimas de madera. La principal fuente de emisiones en estas empresas se da

\footnotetext{
${ }^{6}$ Sand blast es un proceso que consiste en la limpieza de una superficie por la acción de un abrasivo granulado expulsado por aire comprimido a través de una boquilla. La limpieza con sand blast es ampliamente usada para remover óxido, escama de laminación y cualquier tipo de recubrimiento de las superficies, y las prepara para la aplicación de un recubrimiento (CAM, 1997).
} 
en el proceso de tratamiento térmico de las tarimas ${ }^{7}$ y en el proceso de corte de la madera. Las principales emisiones contaminantes identificadas en el informe de auditoría incluyen emisiones al aire $\left(\mathrm{NO}_{2}, \mathrm{CO}\right.$ y PST) y generación de aceites residuales. El cumplimiento ambiental respecto de las autorizaciones aplicables a estas empresas es nulo (autorización de impacto ambiental y el registro estatal de emisiones a la atmósfera). El cumplimiento en el resto de los rubros es bajo (impacto ambiental, emisiones a la atmósfera, gases de combustión, partículas suspendidas totales y residuos industriales no peligrosos). Por lo anterior, esta rama presentó un PP bajo y un PC bajo.

\section{Imprenta}

Las emisiones generadas en esta actividad son originadas en los procesos de revelado de negativos, limpieza de placas y limpieza de la prensa para impresión, en los que se manejan sustancias químicas como tintas y alcohol. Esta rama presenta un PP medio, las principales emisiones son COV, olores, gases y aguas residuales potencialmente contaminantes. El cumplimiento ambiental en esta rama fue nulo tanto en autorizaciones como en el resto de los rubros (emisiones a la atmósfera y residuos tanto peligrosos como no peligrosos). En síntesis, esta rama presentó un PP medio y un PC nulo.

Estos resultados comprueban, por un lado, una generalizada falta de cumplimiento ambiental en las Pyme que entraron al PAA; por otro, señalan las diferencias en los perfiles de contaminación que pueden presentar estas empresas entre diferentes ramas y hacia dentro de cada rama y que permiten identificar posibles ramas prioritarias en el control de la contaminación con respecto a otras.

\section{Conclusiones}

Ante la escasez de datos sobre la contaminación que generan las Pyme, la aplicación de la AA como herramienta principal de programas voluntarios o de autorregulación ambiental proporciona una alternativa para que las Pyme y las autoridades locales competentes tengan una aproximación sobre los problemas de contamina-

\footnotetext{
${ }^{7}$ Este proceso es un requisito fitosanitario de carácter internacional para la exportación de embalajes de maderas, que en México entró en vigor a partir del año 2005 con la publicación de la NOM-144-SEMARNAT-2004 impulsadas por la expedición de las "directrices para reglamentar el embalaje de madera utilizado en el comercio internacional” (Norma NIMF15).
} 
ción que genera este segmento de la economía e identificar las oportunidades para mejorar su cumplimiento ambiental.

Los perfiles ambientales de las Pyme analizadas dentro del PAA muestran un nulo cumplimiento ambiental en combinación con distintos niveles de contaminación. Las ramas participantes con mayor nivel de contaminación resultaron ser alimentos, productos metálicos y remanufactura automotriz. Las diferencias entre las ramas señalan la necesidad de políticas ambientales específicas y el establecimiento de prioridades.

El diseño de iniciativas o estrategias que combinen las ventajas de la AA con otros instrumentos ambientales como los incentivos económicos, indicadores ambientales, ecoetiquetas, etc., es un elemento fundamental para pasar de un diagnostico de la situación ambiental a un proceso de mejora continua del desempeño ambiental de las Pyme.

El uso de instrumentos basados en información como el diseño de una página de Internet que contenga guías de autoevaluación y además ofrezca estudios de caso sobre mejores prácticas o innovaciones basados en la experiencia de las Pyme locales, nacionales o internacionales, es otra opción.

La implementación de un enfoque de redes es una alternativa que puede ser considerada para mejorar el PAA Mexicali, sobre todo al considerar que en la ciudad hay diversas Pyme que son proveedoras de industrias de gran tamaño (maquiladoras) que cuentan con avances en su gestión ambiental (ISO 14001 o Industria Limpia); la colaboración entre universidades, otras Pyme, el gobierno y otras organizaciones también resulta un nicho importante por explorar. Las universidades, por ejemplo, pueden ofrecer programas de servicio social en los que los estudiantes sean capacitados y sirvan de asesores para auditar ambientalmente a un mayor número de Pyme u ofrecer opciones de mejoras de procesos o tecnologías, innovación y gestión ambiental aplicables a empresas específicas.

Sin duda, se requiere de una mayor participación de las Pyme en este tipo de programas para tener un diagnóstico más completo sobre la contribución de este sector a la contaminación en la ciudad; asimismo, la realización de estudios más detallados para corroborar los efectos que puede causar la contaminación de dichas empresas a la población vecina y al ambiente según la distribución geográfica que presentan. El mayor número de datos que caractericen la contaminación generada 
por las Pyme en una ciudad puede permitir una redefinición de objetivos de control de contaminación, ya sea por rubro (aire, agua, suelo), o en su caso una redefinición de la población objetivo, por rama, por localización o concentración.

El establecimiento por parte de las autoridades correspondientes de iniciativas o estrategias efectivas y el uso de instrumentos para mejorar el cumplimiento ambiental de las Pyme requiere considerar los distintos factores limitantes que pueden presentar cada uno de los subgrupos de Pyme localizadas en un mercado local. Del mismo modo, hay que considerar que los diversos instrumentos disponibles para mejorar el cumplimiento ambiental no son aplicables a todo tipo de Pyme y por todo tipo de autoridad local, por lo que no existe una única solución óptima para el total de estas empresas. Una combinación de instrumentos que funcionen como incentivos (carrots) y sanciones (sticks) efectivas puede llevar a mejores resultados.

\section{Referencias}

Brío, Jesús Ángel y Beatriz Junquera (2002). Gestión medioambiental en la PYME: Consideraciones para las políticas públicas. Información Comercial Española, ICE: Revista de Economía, 798: 191-206.

Bojórquez-Tapia, Luis Antonio y Ofelia García (1995). Aspectos metodológicos de la auditoría ambiental. En Pemex: ambiente y energía. Los retos del futuro. UNAM-PEMEX

Carmona, Maria del C. (1995). Aspectos jurídicos de la Auditoría Ambiental en México. En Pemex: ambiente y energía. Los retos del futuro. UNAM-PEMEX.

CAM (1997). Manual de minimización, tratamiento y disposición. Concepto de manejo de residuos peligrosos e industriales para el giro metalmecánico, Comision Ambiental Metropolitana y Sociedad Alemana de Cooperación Técnica (GTZ), México. 
Cepis, EPA y BID (2000). Prevención de la contaminación en la pequeña y mediana industria. Guía de criterios y conceptos básicos (volumen I). Recuperado el 16 de diciembre de 2009. Disponible en: http://www.cepis.org. pe/eswww/fulltext/resipeli/preven/web/index.htm

Domínguez, Lilia (2003). Necesidades de bienes y servicios ambientales en las micro y pequeñas empresas: el caso mexicano. Serie medio ambiente y desarrollo, CEPAL/Sociedad Alemana de Cooperación Técnica. 61.

Firman plan de regulación ambiental (2005, 6 de agosto). La crónica. Recuperado el 5 de septiembre de 2009. Disponible en: http://www.lacronica.com/EdicionImpresa/EjemplaresAnteriores/BusquedaEjemplares.asp?numnota=38 $5865 \&$ fecha $=06 / 08 / 2005$

Gunningham, Neil (2002). Regulating small and medium sized enterprises. Journal of Environmental Law. 14: 3-32.

Hillary, Ruth (1995). Small firms and the environment: a groundwork status report, Groundwork, Birmingham, UK.

(ed.) (2000). Small and medium-sized enterprises and the environment: business imperatives, Greenleaf Publishing Ltd, Sheffield.

Hobbs, Jonathan (2000). Promoting cleaner production in small and medium-sized enterprises. En Hillary, Ruth (Ed.): Small and medium-sized enterprises and the environment. Business imperatives. Greenleaf Publishing Limited, Sheffield: $148-157$.

INEGI, Semarnap, Cenica y PNUD (2000). Elementos para un proceso inductivo de gestión ambiental de la industria, México. Recuperado el 25 agosto de 2008. Disponible en: http://www.ine.gob.mx

INEGI (2004). Censos económicos de México. Aguascalientes: INEGI

Kent, Lawrence (1991). The relationship between small enterprises and environmental degradation in the developing world (with emphasis on Asia), Prepared for the Office of Small, Micro and Informal Enterprises, USAID, Estados Unidos. 
La Secretaría de Protección al Ambiente del estado y Canacintra Mexicali certificaron 40 empresas en el programa autorregulación ambiental (2007, 14-18 de mayo). Boletín informativo SEDECO, 95. Recuperado el 30 de Noviembre de 2009. Disponible en: http://www.bajacalifornia.gob.mx/sedeco/boletin/ b95/b2.htm

Ley General de Equilibrio Ecológico y Protección al Ambiente. Reforma publicada en el Diario Oficial de la Federación el 5 de julio de 2007.

Meritt, J. Quentin (1998). EM into SME won't go: attitudes, awareness and practices in the London Borough of Croydon. Business Strategy and the Environment. 7: 90-100.

Monkhouse Claire, David Wilkinson, Martina Herodes, Peter Hjerp (2006). Environmental compliance assistance for SMEs: analysis of specific initiatives at national and local level and identification of best practices. Institute for Environmental European Policy. Recuperado el 20 diciembre de 2009 . Disponible en: http://ec.europa.eu/environment/sme/pdf/ieep_en.pdf

OCDE (2005). OECD SME and Entrepreneurship Outlook. 2005 Edition. OCDE.

ONU (1992). Agenda 21. Recuperado el 12 de abril de 2010. Disponible en: http:// www.un.org/esa/dsd/agenda21_spanish/

Parker, Craig M., Janice Redmond y Mike Simpson (2009). A review of interventions to encourage SMEs to make environmental improvements. Small and Medium Enterprise Research Centre Papers, paper 2. Recuperado el 5 de junio de 2010. Disponible en: http://ro.ecu.edu.au/rsmerc/2

Pimenova, Polina y Rita van der Vorst (2004). The role of support programmes and policies in improving SMEs environmental performance in developed and transition economies. Journal of Cleaner Production. 12, 6: 549-559.

Revell, Andrea (2002) Ecological modernisation of small firms in Japan, Environmental economics and policy studies. 5: 291-317. 
Romo, David (2005). Políticas e instrumentos para mejorar la gestión ambiental en las pymes y promover la oferta de bienes y servicios ambientales: el caso mexicano. Serie medio ambiente y desarrollo, CEPAL/Sociedad Alemana de Cooperación Técnica. 95.

Sánchez, Luis E. (2002). Auditorías ambientales. En Repetto Fernando y Claudia Karez (editores) Notas de clase del II Curso Internacional de aspectos geológicos de protección ambiental. UNESCO para América Latina y el Caribe. Recuperado el 5 de junio de 2010. Disponible en: http://www.unesco.org. uy/geo/campinaspdf/campinasprimeras.pdf

Secretaría de Planeación y Finanzas. (2008), Avance del tercer trimestre del ejercicio fiscal 2007. Baja California, México: Gobierno de Baja California.

Secretaría de Protección al Ambiente (s. f.), Programa de Autorregulación Ambiental [Folleto]. Baja California, México: Gobierno de Baja California.

Secretaría de Protección al Ambiente (2006). Decreto del ejecutivo del estado que exime del pago de derechos ante la Secretaría de Protección al Ambiente, Periódico Oficial del Estado de Baja California, 17 de noviembre.

Starkey, Richard (ed.) (1998). Environmental management tools for SMEs: a handbook. Environmental Issues Series. Denmark: European Environment Agency.

Tilley, Fiona (1999). Small-firm environmental strategy: The UK experience. Greener Management International. 25. Greenleaf Publishing.

UNEP (2003). Networks and tools for SMEs. UNEP Industry and Environment. $26,4 . q$ 
